\title{
From spin models to lattice QCD - the scientific legacy of Peter Hasenfratz
}

\author{
Urs Wenger* \\ Albert Einstein Center for Fundamental Physics \\ Institute for Theoretical Physics \\ University of Bern \\ Sidlerstrasse 5 \\ CH-3012 Bern \\ Switzerland \\ E-mail: wengeraitp.unibe.ch
}

This is a transcript of my conference talk in remembrance of Peter Hasenfratz who deceased earlier in 2016. One of Peter's many important contributions to the lattice community has been the initiation of the first lattice conference at CERN in 1982. From among his many important ideas, which Peter contributed to our field, I choose to discuss three in some detail and show how they are influencing the subject today.

34th annual International Symposium on Lattice Field Theory

24-30 July 2016

University of Southampton, UK

${ }^{*}$ Speaker. 


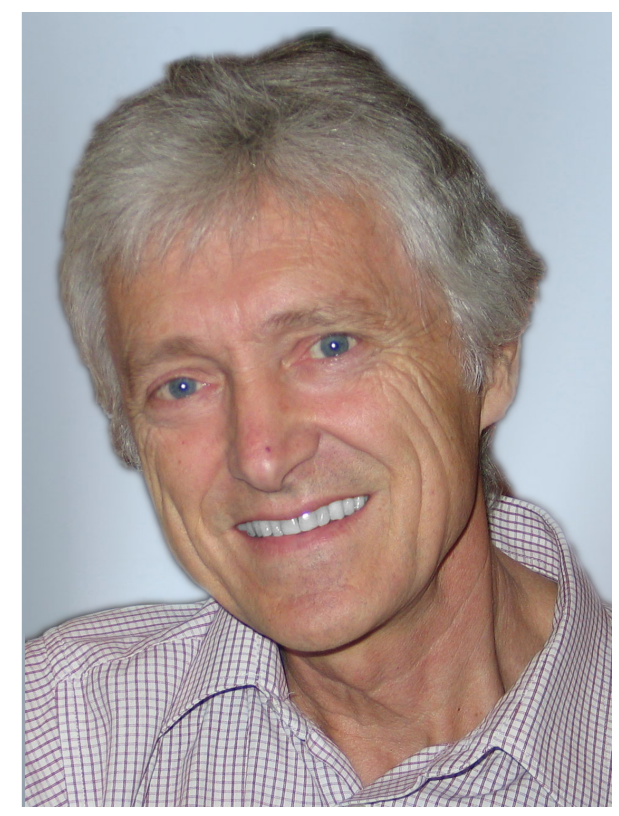

Péter Hasenfratz 1946 - 2016

It is with great sorrow that we have to accept the fact that Peter Hasenfratz deceased earlier this year on April 9, 2016 at the age of 69 as a consequence of his suffering from Alzheimer's disease. Our condolences go out to his family, in particular also to his sister and our colleague Anna, and to his friends and colleagues.

Peter was a very gentle and extremely modest person and he had a very generous personality. His generosity did not only reach out to his family, but also to his friends and colleagues. His kindness is very nicely captured by a quote of an old friend of Peter: "If I would not know Péter's profession, I would have guessed he must be a pediatrician". Peter's softness is also reflected in a statement by Peter himself, which he once mentioned to me: "If I would not have become a scientist, I would be a poet". I think this quote expresses quite well his personality, but also his special way how he looked at nature. Nevertheless, despite his poetic approach to physics, he would tolerate only the highest scientific standards and was very tough when it came to discussing physics. - We are sad to have lost an extraordinary person and scientist.

Before appreciating some of Peter's scientific achievements, let me briefly recall his CV. Peter was born in Budapest on September 22, 1946. After his B.Sc. in Physics in 1971 at Eötvös University, Budapest, he continued studying physics and received his Ph.D. degree in 1973, also from Eötvös University. Subsequently, he became a member of the Central Research Institute for Physics (CRIP) in Budapest, before taking a postdoc position from 1975 to 1976 in Utrecht, working together with Gerard 't Hooft. After two years back at CRIP he became a postdoc at CERN in 1979 and was promoted to a staff member at the Theory Division in 1981. Only three years later he got an associate professorship at the Institute for Theoretical Physics (ITP) of the University of Bern in 1984 and was promoted to full professor in 1991. With intermittent sabbatical stays in Santa Barbara, Boulder, Boston and CERN he stayed at the ITP until 2011 when he retired.

\section{Peter Hasenfratz' scientific contributions}

It is difficult to summarize Peter's vast scientific contributions in just a short amount of time, and it is even more difficult to acknowledge it in terms of numbers. Peter has over 125 published articles which by today's standards does not seem a lot. I think this simply reflects the fact that his publications only followed very high quality standards which were difficult to satisfy, not least by himself. He would publish only when he considered the results worthwhile and significant. Many of the publications are in fact proceedings of plenary talks, review articles, and lecture notes. Not suprisingly, he was an excellent academic teacher, not only during his lectures at the university, but also at numerous international schools. As a student it was difficult not to get infected by 
his enthusiasm about quantum field theory (QFT) in particular and particle physics in general. Consequently enough, he has also been an advisor to a total $14 \mathrm{Ph} . \mathrm{D}$. students.

One particular scientific contribution is actually of sociological nature. In 1982 Peter initiated the first lattice conference as a workshop at CERN. In the unimaginable times without internet, he issued handwritten personal invitations to all participants. In this way, he put the seed for a new scientific community, which over the years has grown into what we experience today at this conference. So far, there have been 33 conferences with several hundreds of participants each - the 34th symposium here in Southampton for example boasts more than 420 participants.

Peter seemed to have an ability to analytically calculate seemingly uncalculable things. Let me here just mention two specific examples which I will discuss in more detail later, the scale parameter of QCD on the lattice on the one hand, and the exact mass gaps in several two-dimensional asymptotically free quantum field theories on the other hand. He was a very creative and original thinker and provided numerous seminal contributions to lattice QFT, such as the concept of Fixed Point (FP) actions, the index theorem, the understanding of lattice chiral symmetry, the proper lattice definition of the chemical potential, and so on and on, just to mention here the concepts which I will refer to later in my talk. Other major research topics to which Peter contributed include the quark bag model, topological excitations, spin models, hopping expansion, Higgs physics (upper bound, top quark condensate, ...), finite size effects from Goldstone bosons, or finite temperature phase transitions in QCD. Higgs physics was a topic Peter was particularly fond of. The picture on the right shows him delivering a plenary talk

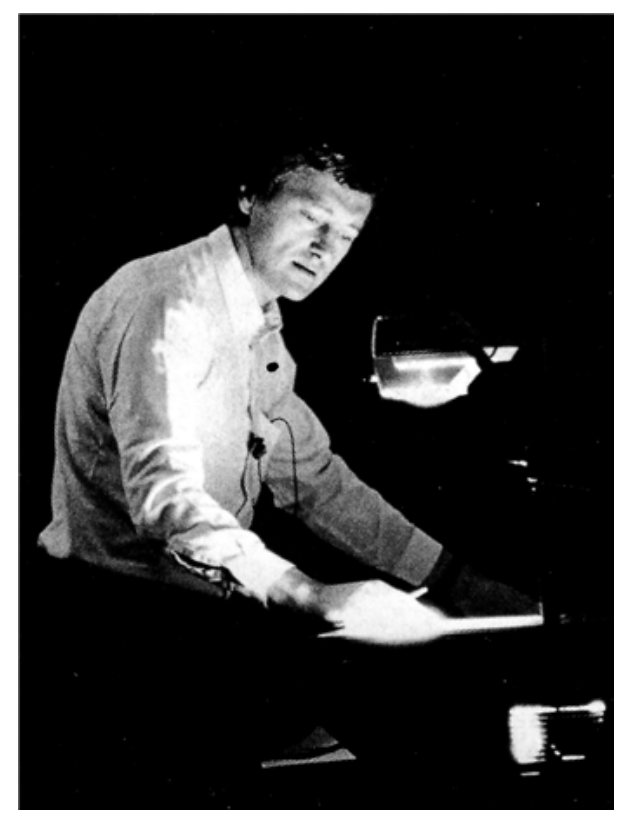

Figure 1: Peter Hasenfratz delivering a plenary talk on the mass limits of the Higgs particle at the lattice conference at Fermilab in 1988 [1] (picture from [2]).

on the mass limits of the Higgs particle at the lattice conference at Fermilab in 1988. Throughout his career he continued to be fascinated by the Higgs mechanism and the underlying field theoretic concepts, and he certainly enjoyed the discovery of the Higgs particle a few years ago.

It would be impossible to cover all of the topics mentioned above - instead I will just focus on three separate topics which I find particularly interesting, not least because they connect to issues which are going to be discussed at this conference. The first topic concerns the connection between the lattice and the continuum, the second concerns the calculation of mass gaps, and the third the renormalization group (RG) and fixed point (FP) actions.

\section{The connection between the lattice and the continuum}

Let us turn back the clock about 35 years into a time when lattice field theory, and in particular numerical calculations, started to come out of its infancy. Peter was very busy as a salesman in the high-energy particle physics community, giving many talks on the lattice approach to field theory 
in general, and QCD in particular. Despite being a salesman, he did never stop to emphasize the shortcomings of these early calculations, to many of which Peter contributed himself, and he kept being very critical towards them. Let me quote in the following a few statements which can be found in his review paper on lattice gauge theories from 1981 [3]: "MC simulations did not help us to obtain a better physical understanding, a deeper insight into the theory." - "Is g small enough? [...] there is reason to worry: the approach to asymptotic scaling might be very slow." - "In spite of the intense work, there is no real progress one can report on." - "The whole program is faced with unexpected and unpleasant difficulties at this moment." - "Clarification is needed." - "One should consider these numbers with some reservations." - "[...] although it is not clear whether every part of the calculation is under control." - Further into 1982 [4] he continued along the same line, always keeping expectations low and advocating a slow and careful approach, instead of a fast and attention-grabbing one: "[...] the reliability of this procedure is really questionable." "I sense a big change concerning the expectations of the physics community. Actually I believe this change is too big." - "Please, have your own healthy doubts [...]. Solving QCD is not so easy." - "[...] admit clearly the defects of our methods and make serious efforts to improve them. This path is less spectacular, but, perhaps, worth following." - All these statements emphasize the carefulness and great diligence which Peter applied to the lattice approach, and I would like you to keep this in mind, when I discuss some of the very latest results from lattice QCD at the end of this section.

In the late seventies and early eighties it was not yet clear whether lattice QCD provides a sensible regularization of continuum QCD. Hence, in order for lattice QCD to be meaning- and useful on a practical level, it was obviously crucial to establish the connection between calculations on the lattice and in the continuum. Lattice QCD, either in the chiral or the quenched limit, contains only the dimensionless coupling $g$ and implicitly the dimensionful lattice spacing $a$ as parameters. For a physical mass $m$, or equivalently a correlation length $\xi$, one therefore has

$$
m=f(g) \cdot \frac{1}{a} \quad \text { or } \quad \xi=h(g) \cdot a .
$$

The continuum limit is reached when $1 / m$ or $\xi \gg a$, i.e. when the lattice system approaches a continuous phase transition. In asymptotically free theories, the limit is straightforward to reach, since the lattice spacing $a \rightarrow 0$ for $g \rightarrow 0$. By changing $a$ and $g$ appropriately towards the continuum limit, physical quantities should become independent of $a$,

$$
\frac{d}{d a} m=0 \quad(a \rightarrow 0),
$$

which is equivalent to say that the theory is renormalizable. The renormalizability uniquely fixes the relation between $a$ and $g$ through the differential equation for $f(g)$,

$$
-f(g)+f^{\prime}(g)\left(a \frac{d}{d a} g\right)=0 \quad \text { where } \quad \beta(g) \equiv a \frac{d}{d a} g=-b_{0} g^{3}-b_{1} g^{5}-\ldots
$$

Hence, every physical quantity on the lattice can be expressed in terms of a single, RG-invariant mass parameter $\Lambda^{\text {latt }}$, e.g. $m=c_{m} \cdot \Lambda^{\text {latt }}$, and the dependence of the scale on the gauge coupling $g$ is 
determined by Eq. (2.1), yielding

$$
\Lambda^{\text {latt }}=\frac{1}{a} e^{-1 / 2 b_{0} g^{2}}\left(b_{0} g^{2}\right)^{-b_{1} / 2 b_{0}^{2}} \cdot\left[1+\mathscr{O}\left(g^{2}\right)\right]
$$

to lowest order in perturbation theory. Analogously, in a continuum renormalization scheme one has

$$
\Lambda=M e^{-1 / 2 b_{0} g(M)^{2}}\left(b_{0} g(M)^{2}\right)^{-b_{1} / 2 b_{0}^{2}} \cdot\left[1+\mathscr{O}\left(g(M)^{2}\right)\right]
$$

where the mass parameter $M$ corresponds to the renormalization scale introduced in the continuum renormalization scheme.

So, in order to set the scale in the lattice theory, and to make sense of it, one better connects the two scales. In 1980 Anna and Peter Hasenfratz calculated this connection [5]:

$$
\Lambda_{\text {Feynman gauge }}^{\mathrm{MOM}}=83.5 \Lambda^{\text {latt }} \quad \text { for } S U(3), \quad \Lambda_{\text {Feynman gauge }}^{\mathrm{MOM}}=57.5 \Lambda^{\text {latt }} \text { for } S U(2) .
$$

The computation involves a rather long and tedious 1-loop calculation of 2- and 3-point functions in lattice perturbation theory. In particular, the calculation provides an explicit demonstration that there are no unwanted divergences and that all noncovariant terms cancel. In that sense, the result truly constitutes a milestone in establishing lattice QCD as a viable and useful regularization of QCD. Anna and Peter were the first to get the relation correct, thereby settling a dispute which was ongoing at the time. It is hard to overestimate the challenge and the difficulty of this calculation, and in fact it took 15 more years until the corresponding 2-loop calculation was completed [6].

Of course, the $\Lambda$ parameter is nonperturbatively defined,

$$
\Lambda=M e^{-1 / 2 b_{0} g(M)^{2}}\left(b_{0} g(M)^{2}\right)^{-b_{1} / 2 b_{0}^{2}} \times \exp \left[-\int_{0}^{g(M)} d x\left(\frac{1}{\beta(x)}+\frac{1}{b_{0} x^{3}}-\frac{b_{1}}{b_{0}^{2} x}\right)\right],
$$

and lattice QCD is the ideal method to relate it nonperturbatively to the low-energy properties of QCD. In fact, the $\Lambda$ parameter is a quantity which nowadays is rather well determined from lattice QCD calculations. In Fig. 2 we show the latest collection of lattice QCD results for the $\Lambda$ parameter in the $\overline{\mathrm{MS}}$ scheme in units of $r_{0}$ from the FLAG report [7]. Obviously, while the results for $N_{f}=0$ and 2 flavour QCD are of no interest from a phenomenological point of view, they certainly are for the theoretical understanding of QCD in general.

Closely related to the $\Lambda$ parameter is the running strong coupling $\alpha_{s}$ at the renormalization scale $M$,

$$
\alpha_{s}(M)=\frac{g^{2}(M)}{4 \pi}
$$

It can for example be determined by measuring a short distance quantity $\mathscr{O}$ at scale $M$ and matching it with the

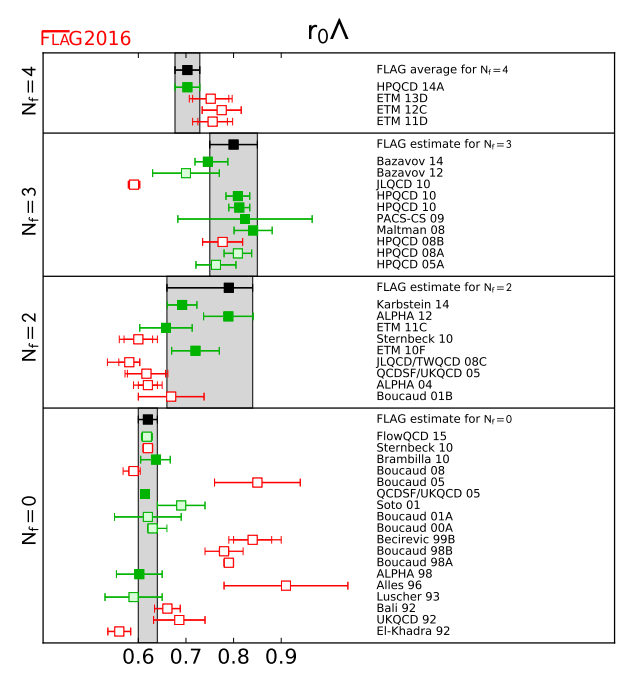

Figure 2: Lattice QCD results for the $\Lambda$ parameter in the $\overline{\mathrm{MS}}$-scheme in units of $r_{0}$ from the FLAG report [7]. 

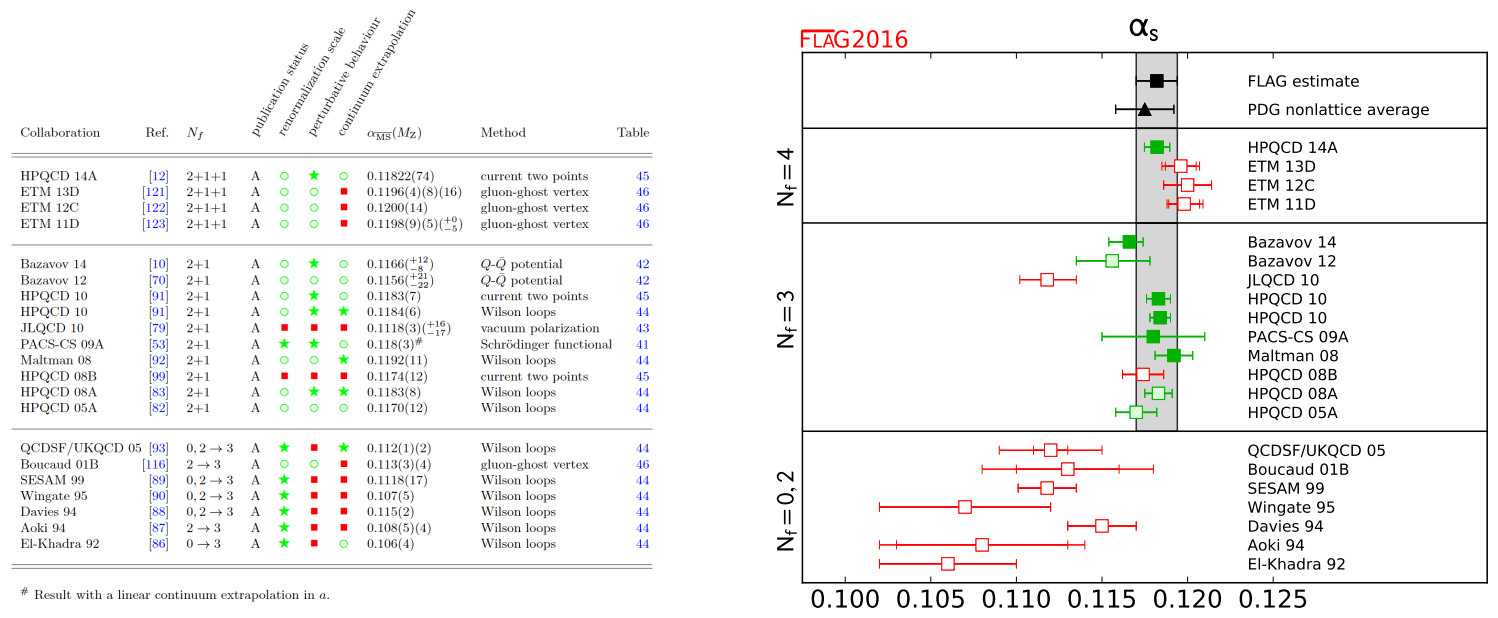

Figure 3: Collection of lattice QCD results for the $N_{f}=5$ strong coupling $\alpha_{\overline{\mathrm{MS}}}^{(5)}\left(M_{Z}\right)$ in the $\overline{\mathrm{MS}}$-scheme at the scale $M_{Z}$ from the FLAG report [7].

corresponding perturbative expansion in terms of the coupling in the $\overline{\mathrm{MS}}$-scheme,

$$
\mathscr{O}(M)=c_{1} \alpha_{\overline{\mathrm{MS}}}(M)+c_{2} \alpha_{\overline{\mathrm{MS}}}(M)^{2}+\ldots
$$

Also for this quantity, lattice QCD calculations are well advanced, as can be seen from the table and plot in Fig. 3. Many collaborations provide values for the $N_{f}=5$ strong coupling $\alpha_{\frac{\mathrm{MS}}{(5)}}\left(M_{Z}\right)$ in the $\overline{\mathrm{MS}}$-scheme at the scale given by the $Z$-boson mass $M_{Z}$, employing a variety of different short distance quantities. It is clear, that a critical assessment of the situation is necessary in order to provide a consistent picture and a reliable estimate of the strong coupling useful for phenomenology. This is exactly what FLAG provides in its review [7]. A careful evaluation indicates that for the strong coupling the dominant source of uncertainty comes from discretization errors and the truncation of continuum and lattice perturbation theory.

It is interesting to compare the FLAG 16 lattice average $\alpha_{\overline{\mathrm{MS}}}^{(5)}\left(M_{Z}\right)=0.1182(12)$ with the values from the 2016 edition of the PDG [8]. They quote $\alpha_{\overline{\mathrm{MS}}}^{(5)}\left(M_{Z}\right)=0.1174(16)$ for an average of all nonlattice results, and $\alpha \frac{(5)}{\mathrm{MS}}\left(M_{Z}\right)=0.1181(11)$ for the world average including the results from the lattice. It is clear from Fig. 4 that the lattice determination by now provides the most precise value. It is gratifying to see that after more than three decades the common effort of the lattice community finally starts to pay off.

In fact, as compared to its 2014 edition, the PDG is now following a more conservative approach for averaging the lattice results, very much in the spirit of FLAG 16, and even more so in

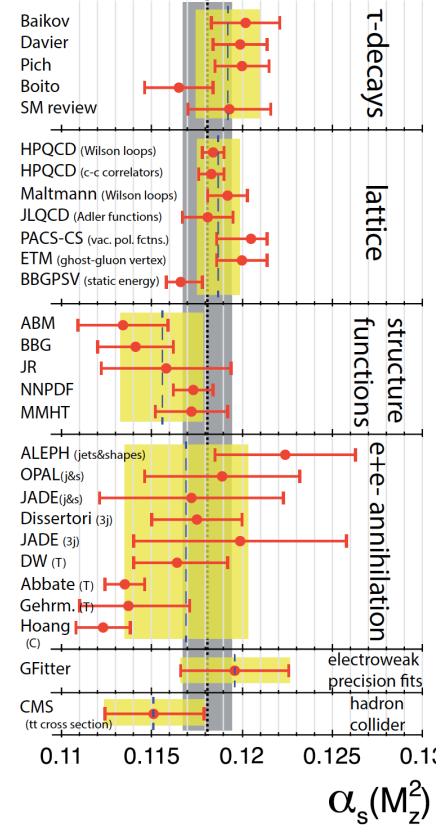

Figure 4: Summary of determinations of $\alpha_{\overline{\mathrm{MS}}}\left(M_{\mathrm{Z}}^{2}\right)$ from the 2016 edition of the PDG [8]. the spirit of Peter. Indeed, it seems that Peter was right 35 years ago, when he advocated to follow a conservative approach, even though it is less spectacular. The conservative approach followed by 


\begin{tabular}{|c|c|c|c|c|c|}
\hline Rererenerec & 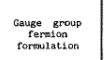 & 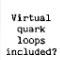 & $\mid$\begin{tabular}{|c|} 
Lattice size \\
No. of itterations
\end{tabular} & The scope of the investization & 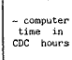 \\
\hline${ }^{111}$ & 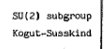 & סו & $\begin{array}{l}8^{8}+8^{8} \cdot 16 \\
4 \text { confs. }\end{array}$ & 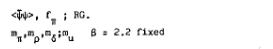 & -10 \\
\hline 12) & 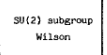 & 10 & $\begin{array}{l}221 \\
\text { o corrs. }\end{array}$ & 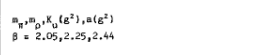 & -100 \\
\hline${ }^{131}$ & 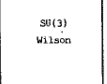 & no & 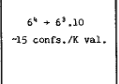 & 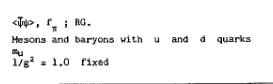 & -100 \\
\hline (4) & 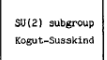 & 100 & $\begin{array}{l}8^{3} .32 \\
8 \text { confs. }\end{array}$ & 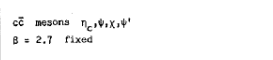 & -2 \\
\hline 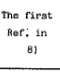 & $\begin{array}{l}\text { sula } \\
\text { vilsoon }\end{array}$ & yrs & 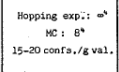 & 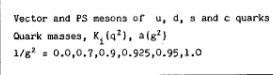 & -5 \\
\hline 151 & $\begin{array}{c}\text { sv(3) } \\
\text { vilison }\end{array}$ & 100 & $\begin{array}{ll}5^{2} \times 10 \\
32 \text { confs, I/k val. }\end{array}$ & 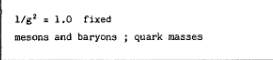 & -100 \\
\hline 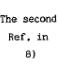 & 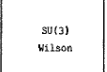 & "o & 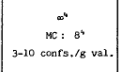 & 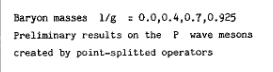 & -10 \\
\hline
\end{tabular}

- Table 2 .

\begin{tabular}{|c|c|c|c|c|}
\hline Referencene & 13i & 8) & 151 & 81 \\
\hline $\begin{array}{l}\text { es and vector } \\
\text { nesen nesses nes }\end{array}$ & $\bar{F}_{p}=800 \mathrm{~s} 100$ Mer & 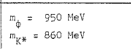 & 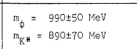 & \\
\hline 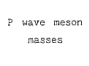 & 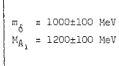 & $\begin{array}{l}\text { no sonss bile results } \\
\text { with pocar operators }\end{array}$ & 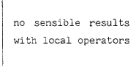 & 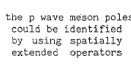 \\
\hline Baryon naseses & 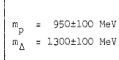 & & 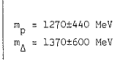 & 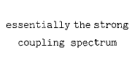 \\
\hline Curark trasese & $\mathrm{N}_{\mathrm{v}, \mathrm{c}, \mathrm{C}} \mathrm{\text {Yever}}$ & 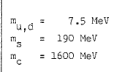 & 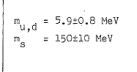 & \\
\hline
\end{tabular}

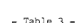

Figure 5: Early tables for critically assessing and summarizing lattice calculations compiled by Peter in 1982 [4].

FLAG requires a critical assessment of the available lattice results, as summarized e.g. in Fig. 3. However, such tables and summaries are not a new invention. In fact, back in 1982 Peter has already provided such tables, cf. Fig. 5, summarizing the properties of various lattice calculations and the corresponding results available at that time [4]. Despite being very critical, Peter was nevertheless always very optimistic, as the following quote by Peter in [3] nicely portrays: "We are able to obtain non-perturbative numbers in a four-dimensional, relativistic, relevant theory. We are proud of it."

\section{The mass gaps}

One of the most difficult problems in a quantum field theory is the determination of the relation between the renormalized couplings from the Lagrangian and the physical masses of the theory, such as for example the nucleon mass in the chiral limit of QCD in units of $\Lambda^{\overline{\mathrm{MS}}}$,

$$
m_{N}=c_{m_{N}} \cdot \Lambda^{\overline{\mathrm{MS}}}
$$

The difficulty lies in the fact that the Lagrangian is defined at short distances (UV-scale), while the masses are parameters at large distances (IR-scale). Surprisingly, there is a family of models where this relation can be found exactly, namely the $\mathrm{O}(N)$ nonlinear sigma models in $d=2$ dimensions. For $N \geq 3$ these integrable models are asymptotically free and contain massive $\mathrm{O}(N)$ isovector multiplets. In 1990 Peter, together with Michele Maggiore and Ferenc Niedermayer, calculated this relation exactly for $N=3$ and 4 [9]:

$$
\begin{array}{ll}
m=\frac{8}{e} \cdot \Lambda^{\overline{\mathrm{MS}}} & N=3, \\
m=\sqrt{\frac{32}{\pi e}} \cdot \Lambda^{\overline{\mathrm{MS}}} & N=4 .
\end{array}
$$


In the same year, Peter and Ferenc extended the calculation to arbitrary $N \geq 3$ [10]:

$$
m=\left(\frac{8}{e}\right)^{1 /(N-2)} \frac{1}{\Gamma(1+1 /(N-2))} \cdot \Lambda^{\overline{\mathrm{MS}}} .
$$

It is interesting to note that at the time, there were over 30 nonperturbative determinations which differed wildly from each other.

While the calculation is rather involved, it is based on a beautiful idea. It starts from the introduction of a chemical potential $h$ coupled to a Noether charge and the observation that the change of the free energy is RG invariant, as is the chemical potential $h$ itself. Then, on the one hand, the free energy can be calculated in perturbation theory in the regime $h \gg m$ where the theory becomes asymptotically free,

$$
f(h)-f(0)=-(N-2) \frac{h^{2}}{4 \pi}\left[\ln \frac{h}{e^{1 / 2} \Lambda_{\overline{\mathrm{MS}}}}+\frac{1}{N-2} \ln \ln \frac{h}{\Lambda_{\overline{\mathrm{MS}}}}+\mathscr{O}\left(\frac{\ln \ln \left(h / \Lambda_{\overline{\mathrm{MS}}}\right)}{\ln \left(h / \Lambda_{\overline{\mathrm{MS}}}\right)}\right)\right] .
$$

On the other hand, since the model is integrable, the free energy can also be calculated by applying the Bethe ansatz, or directly from the $S$-matrix,

$$
f(h)-f(0)=-\frac{m}{2 \pi} \int \cosh \theta \varepsilon(\theta) d \theta
$$

with $\varepsilon(\theta)$ fulfilling a specific integral equation [10]. One can then use a generalized WienerHopf technique to express the integral equation in terms of $\ln h / m$, again in the regime $h \gg m$, and read off the mass $m$ in terms of $\Lambda_{\overline{\mathrm{MS}}}$ by comparing the expressions obtained from the two approaches. The same idea can be applied to other quantum field theories. Its application yields for example also the exact mass gap in the Gross-Neveu model [11] and in the $d=2+1$ dimensional antiferromagnetic Heisenberg model at low temperatures [12, 13].

The reason why I dwell on this in some detail is due to the fact that the idea to couple a chemical potential to a conserved charge and to calculate the corresponding change in the free energy is very general. In fact, it is relevant and in use even today. One interesting, very recent application is for matching chiral Lagrangians for QCD with different regularizations [14, 15]. The calculation is related to the QCD rotator in the $\delta$-regime where $m_{\pi} L_{s} \ll 1$ and $F_{\pi} L_{s} \gg 1$ [16] and provides a promising new way to determine the low-energy constants of QCD [17] by introducing an infrared cutoff through a finite spatial box size $L_{s}$ and then studying the finite size scaling of the spectrum in the chiral limit. More precisely, the chiral Lagrangian for massless 2-flavour QCD has a $\mathrm{SU}(2) \times \mathrm{SU}(2) \simeq \mathrm{O}(4)$ symmetry and the general $\mathrm{O}(N)$ spectrum is given by a quantum mechanical rotator

$$
E(l)=l(l+N-2) / 2 \Theta \quad l=0,1,2, \ldots
$$

where $\Theta=F^{2} L_{S}^{3}$ is the moment of inertia of the rotator [16]. The next-to-leading (NLO) term of the expansion in $1 /\left(F^{2} L_{s}^{2}\right)$ has been calculated by Peter and Ferenc some time ago [18], while the NNLO terms were obtained more recently in the dimensional regularization (DR) scheme [17] and on the lattice [19]. Finally, the very recent, heroic calculation by Niedermayer and Weisz [14, 15] makes use of the same idea and thereby connects the couplings in the two regularizations of the 
effective theory, i.e. it converts the expressions for physical quantities obtained in the lattice scheme to the corresponding ones in the DR scheme. In particular, it relates the finite-volume mass gap on the lattice to the DR scheme.

\section{The renormalization group and FP actions}

Peter had a very deep appreciation and understanding of the Wilson renormalization group (RG) description of quantum field theories. In this context, he always emphasized the viewpoint that lattice gauge theory is just a statistical system, albeit a rather unusual one due to the local gauge invariance, and that the critical limit corresponds to the continuum limit of the corresponding quantum field theory, as illustrated in Fig. 6 from one of Peter's lecture notes [20]. Since the

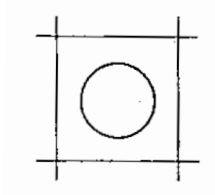

strong coupling

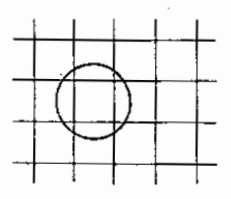

intermediate coupling

$g$ is decreasing

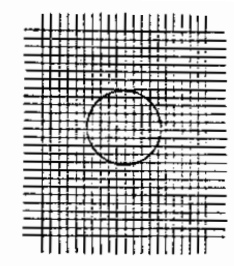

weak coupling

Figure 6: The continuum limit as the critical limit of a statistical lattice system where the lattice spacing becomes small compared to the characteristic physical distances. Figure from [20].

lattice provides a fully nonperturbative description of the phase transition, the continuum physics is recovered in the lattice system at long distances. Close to the phase transition one can integrate out the variables describing the short distance lattice physics and obtain an effective action for the relevant long distance variables in terms of the effective couplings $\left\{K_{\alpha}\right\}$,

$$
\left\{K_{\alpha}^{(1)}\right\} \quad \stackrel{\mathrm{RG}}{\longrightarrow}\left\{K_{\alpha}^{(2)}\right\} \quad \stackrel{\mathrm{RG}}{\longrightarrow} \cdots \stackrel{\mathrm{RG}}{\longrightarrow}\left\{K_{\alpha}^{(n)}\right\} \quad \stackrel{\mathrm{RG}}{\longrightarrow} \cdots .
$$

The sequence of RG transformations might have a fixed point (FP),

$$
\left\{K_{\alpha}^{*}\right\} \quad \stackrel{\mathrm{RG}}{\longrightarrow}\left\{K_{\alpha}^{*}\right\}
$$

In particular, one is interested in a FP where the correlation length of the system is $\xi=\infty$. For gauge theories, the RG transformations are complicated due to the requirement of gauge invariance, but once this is fulfilled, the RG transformations provide the basic starting point in expecting renormalizability and universality along the renormalized trajectory (RT) of the lattice system, cf. Fig. 7 taken from [20].

The fact that the lattice provides a fully nonperturbative description of the RG flow of the couplings and the corresponding FP structure becomes again important today for investigations of quantum field theories beyond the Standard Model (BSM), as also discussed extensively at this lattice conference. It should also serve as a warning for such investigations. Some of the BSM theories are expected to possess a conformal FP, however, an IR FP is in general not perturbative 


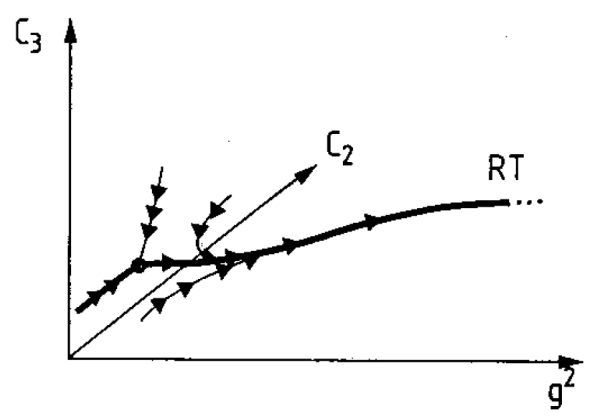

Figure 7: Renormalized trajectory (RT) in the parameter space of gauge couplings. It comes out of the critical hyperplane $g=0$ and attracts all the flow lines starting in the neighbourhood of the fixed point. Figure from [20].

and perturbative intuition could therefore well be misleading. I think it is important to keep this in mind when interpreting some of the results from the numerical BSM calculations dicsussed at this conference, cf. the plenary talk [21].

Back in the early eighties, the application of RG ideas to lattice gauge theory inspired the investigation of perturbative and nonperturbative improvement of lattice actions by making use of approximations to the FP, as e.g. illustrated in the left plot of Fig. 8 taken from Peter's lecture notes from 1983 [20]. While the concept of "(quantum) perfect" actions was already known then, it would take 10 years to make it into something more specific. In 1993 Peter and Ferenc Niedermayer realized that for asymptotically free theories the path integral defining the FP for RG transformations reduces to classical saddle point equations,

$$
S_{G}^{\mathrm{FP}}[V]=\min _{\{U\}}\left[S_{G}^{\mathrm{FP}}[U]+T_{G}[V, U]\right]
$$

where $S_{G}^{\mathrm{FP}}$ is the FP gauge action, $T_{G}$ a blocking kernel defining a RG transformation, while $U, V$ are the gauge fields on the fine and coarse lattices, respectively, related by the RG transformation [22]. The FP Dirac operator is similarly defined by

$$
D^{\mathrm{FP}}[V]^{-1}=R[V]+\omega[U] \cdot D^{\mathrm{FP}}[U]^{-1} \cdot \omega[U]
$$

where $\omega$ defines a blocking kernel for the fermion fields. It can then be shown that the action $\beta S_{G}^{\mathrm{FP}}+\bar{\psi} D^{\mathrm{FP}} \psi$ is classically perfect, i.e. it has no lattice artefacts on field configurations satisfying the equations of motions. For each gauge field configuration on the lattice, the minimizing gauge field $U(x, V)$ defines a FP field in the continuum which induces some remarkable properties on the lattice gauge fields. Under certain conditions, all symmetries of the continuum are well defined on the lattice [23] and the FP field allows for a representation of the corresponding infinitesimal transformations on the lattice according to

$$
V_{n} \stackrel{\text { minimize }}{\longrightarrow} U(x, V) \stackrel{\text { transform }}{\longrightarrow} U^{\varepsilon}\left(x^{\varepsilon}, V\right) \stackrel{\text { block }}{\longrightarrow} V_{n}^{\varepsilon} .
$$

Similar considerations can be made for the fermion fields [23]. In particular, the procedure in principle also provides a possibility to define supersymmetric algebras on the lattice. The fact that 

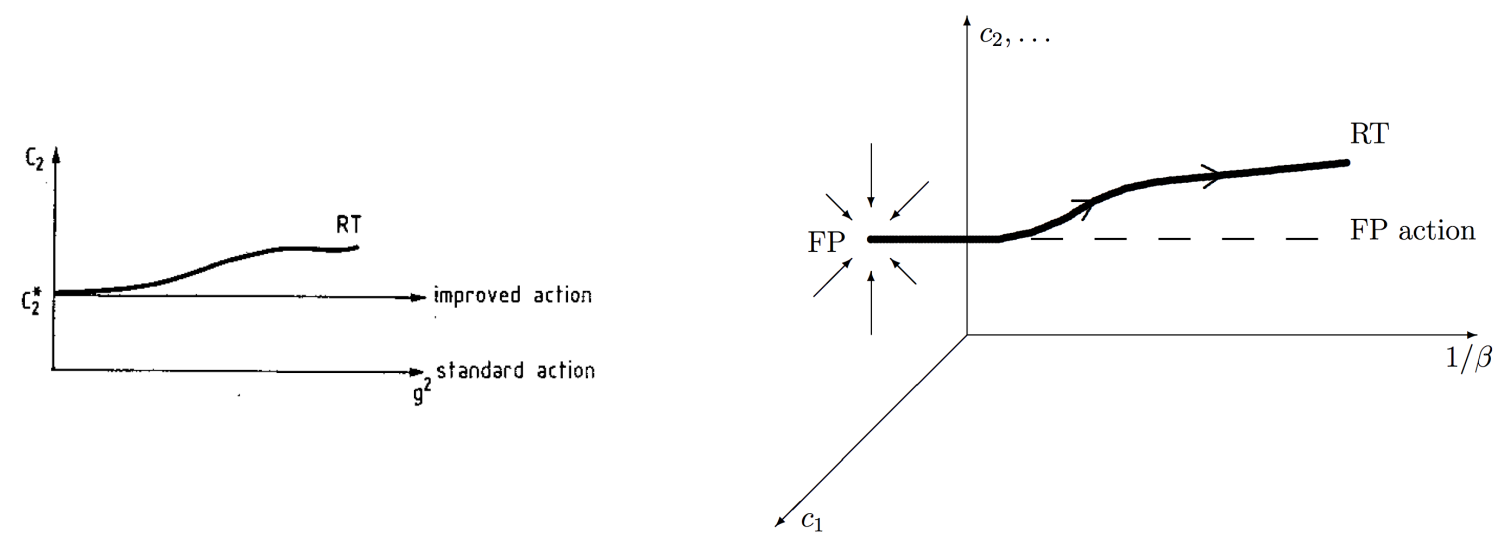

Figure 8: Renormalized trajectory in the parameter space of (gauge) couplings and the improved actions based on an approximation of the FP (left plot) or the exact FP (right plot). The left plot is from 1983 [20], the right plot from 1993 [22].

the FP equations define a scheme to match coarse and fine lattice configurations could also be useful in recent attempts to delay the onset of topological critical slowing down in today's lattice QCD simulations by constructing multi-scale Monte-Carlo update algorithms. One effort in this direction [24, 25], in which the FP action approach could be very useful, is in fact discussed in a plenary talk at this conference [26].

Then, in 1997 Peter made a truly groundbreaking observation [27]. While travelling to a summer school Peter looked through a pile of old preprints which he picked up while tidying up his office. A paper by Ginsparg and Wilson [28] grabbed his attention and he realized that the FP Dirac operator $D^{\mathrm{FP}}$ fulfills the nowfamous Ginsparg-Wilson relation

$$
D \gamma_{5}+\gamma_{5} D=D \gamma_{5} D
$$

The relation is derived from RG transformations applied to free fermions. Any solution of the relation avoids the NielsenNinomyia no-go theo-

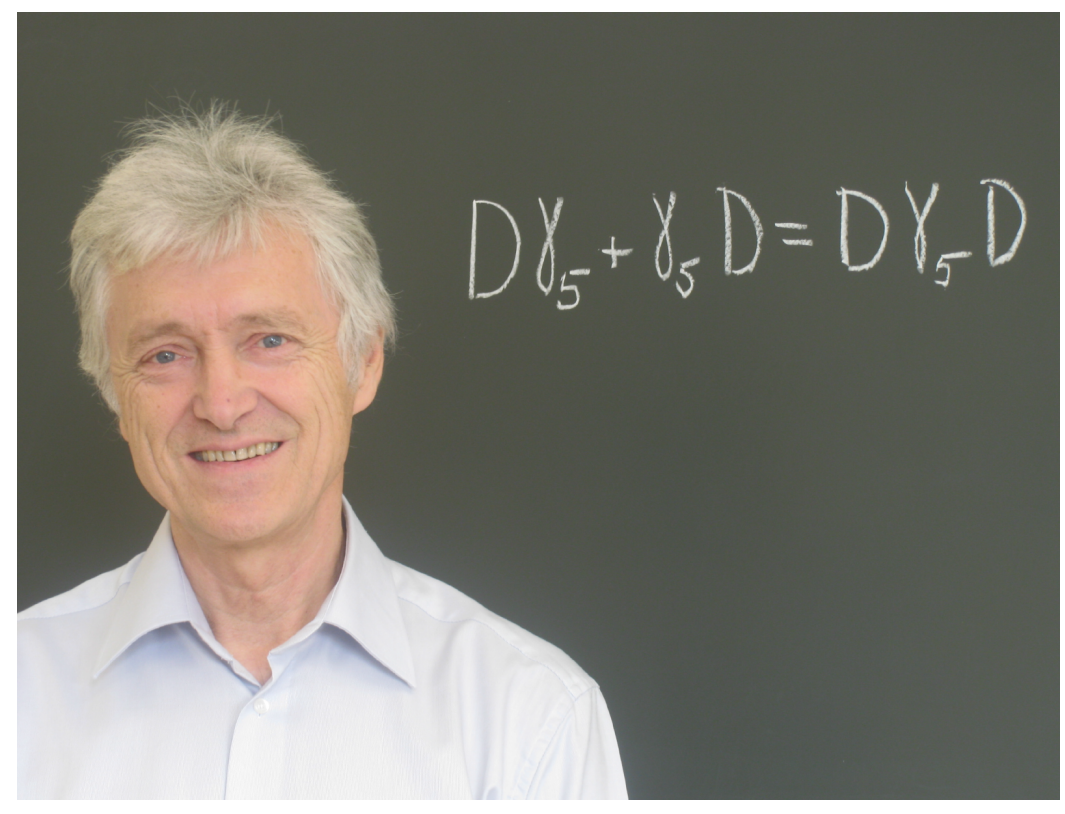
rem, implies the correct triangle anomaly and the validity of all the soft-pion theorems on the lattice [27]. Peter's crucial observation then was that the FP Dirac operator $D^{\mathrm{FP}}$ constitutes a solution for the interacting theory. As a consequence of the Ginsparg-Wilson relation there is no tuning, no mixing and no current renormalization necessary for the FP Dirac operator on the lattice [29]. 
The observation set off an avalanche of developments. This is best visualized by looking at the citation history of the Ginsparg-Wilson paper [28], cf. Fig. 9. It is fascinating to see how the interest in the paper exploded after Peter's rediscovery in 1997 - with 985 citations it is by now the second most cited paper cited by the hep-lat archive [30]. It is impossible to describe in detail the revolution which followed, so let me just mention a few key developments on the theoretical side: the exact index theorem in QCD on the lattice [31]; the overlap operator as another solution to the Ginsparg-Wilson relation [32]; the exact chiral symmetry on the lattice [33]; Abelian chiral gauge theories on the lattice $[34,35]$; the axial anomaly and topology [36, 37, 38, 39]; the chiral Jacobian on the lattice [40, 41]; lattice supersymmetry [42]; and so on and on.

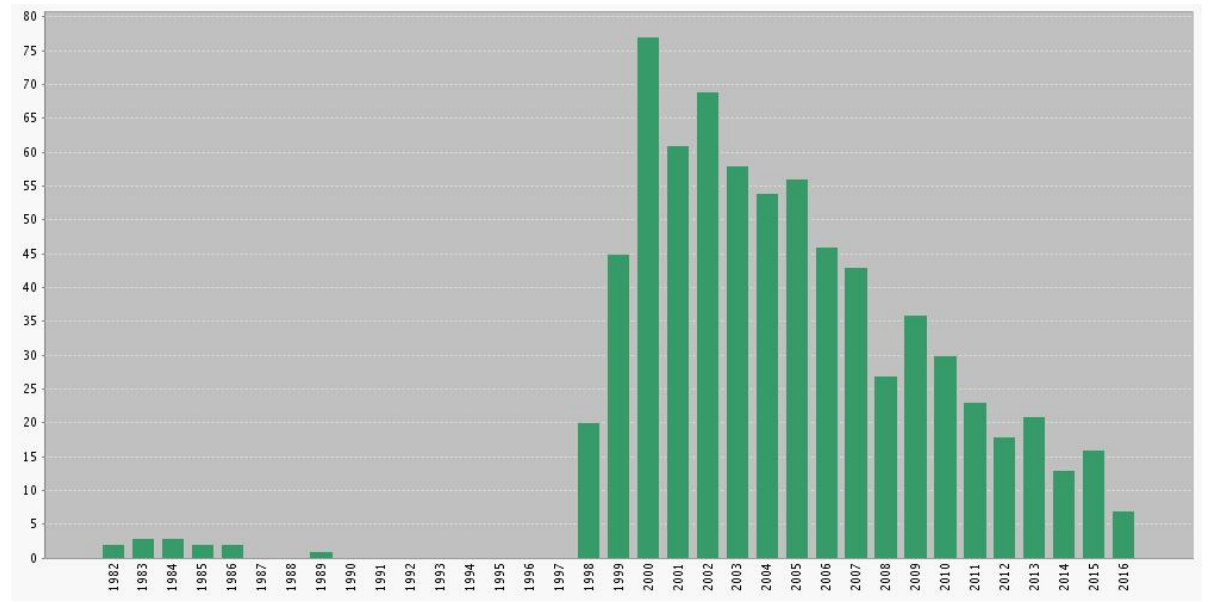

Figure 9: Citation history of the Ginsparg-Wilson paper [28].

Even today, the implications for phenomenological and theoretical applications can hardly be overestimated, and as evidence I just refer to the various parallel sessions such as "Weak decays and matrix elements", "Chiral symmetry", "Theoretical developments", with several talks related to or based on chiral symmetry on the lattice in some way or another.

In order to close this section, let me make a couple of remarks which I find particularly intriguing in the context of chiral symmetry regularized on the lattice. Firstly, the possibility to realize exactly massless fermions on the lattice constitutes a true solution of a hierarchy problem, a fact which is often unappreciated outside the lattice community. Secondly, FP fermions on the one hand and overlap/domain wall (DW) fermions [43, 44, 45, 46] on the other hand derive from two completely different approaches, yet they both fulfill the Ginsparg-Wilson relation. Is there a connection between the two formulations, and if yes, how are they related? While the FP operator is motivated from RG considerations and therefore has a very specific physical meaning, the overlap/DW operator has a less obvious physical interpretation. Rather, the overlap/DW operator can more easily be understood from an algebraic viewpoint. More precisely, the Ginsparg-Wilson relation is a specific form of an algebraic Riccati equation, the solution of which naturally involves the sign function as it also appears in the overlap/DW operator. Thirdly, the chiral transformation on the lattice makes use of the gauge field dependent chiral projectors $\hat{P}_{ \pm}=1 / 2\left(1 \pm \hat{\gamma}_{5}\right)$ with $\hat{\gamma}_{5}=\gamma_{5}(1-D)$. They are particularly important in the context of defining chiral gauge theories on the lattice, a problem which remains to be of high interest and is in fact also discussed in a plenary 
talk at this lattice conference, cf. [47] and references therein to the corresponding lattice talks. On the one hand, the chiral projectors are responsible for the necessary asymmetry between fermions and anti-fermions, on the other hand they also break $C P$ symmetry [48]. The $C P$ breaking is an unwanted feature, the role of which is still not well understood.

\section{Closing remarks}

After my recollection of just three out of many of Peter's scientific contributions, it is important to realize that Peter's legacy is much more than his outstanding scientific achievements. It goes far beyond what he has taught us about quantum field theories on and off the lattice. As researchers we are all curious and strive for the unknown, but Peter taught us what it means to go one step further: do look close and careful, be very critical - and do question even the presumably well-known and the commonly accepted. This will inevitably lead to surprising results, new insights and creative ideas. Of course this is all said easily and quickly - it distinguishes Peter that he was indeed able to follow this path and operate in such a perfect way. The careful and conservative path is usually not very spectacular, but it is certainly much more enduring and lasting, and, as Peter pointed out, worth following. It will sustain and support the lattice community much better in the long term by gaining and keeping a high regard and esteem from outside our community.

One of Peter's legacy obviously is the fact that we all meet once a year to discuss our progress, exchange the latest insights and results, and get excited about new developments. I am very happy to see that this legacy is carried on. Looking into the audience I see many young faces among the very many participants, so I am very optimistic that this legacy will continue for a very long time I am certain that this would make Peter very happy.

I would like to thank Anna Hasenfratz, Kieran Holland, Ferenc Niedermayer and Uwe-Jens Wiese for helpful discussions during the preparation of this talk.

\section{References}

[1] P. Hasenfratz, Upper bound on the Higgs meson mass, Nucl. Phys. Proc. Suppl. 9 (1989) 3-17.

[2] CERN Courier, MEETING Lattice 88, International Journal of High Energy Physics 29, Number 2 (1989) 21-23.

[3] P. Hasenfratz, Lattice gauge theories, in 1981 EPS International Conference on High-Energy Physics Lisbon, Portugal, July 9-15, 1981, p. 0619, 1981.

[4] P. Hasenfratz, Hadron spectrum calculations in lattice QCD, in 6th Johns Hopkins Workshop on Current Problems in Particle Theory Florence, Italy, June 2-4, 1982, pp. 1-14, 1982.

[5] A. Hasenfratz and P. Hasenfratz, The Connection Between the Lambda Parameters of Lattice and Continuum QCD, Phys. Lett. B93 (1980) 165.

[6] M. Lüscher and P. Weisz, Computation of the relation between the bare lattice coupling and the MS coupling in SU(N) gauge theories to two loops, Nucl. Phys. B452 (1995) 234-260, [hep-lat/9505011].

[7] S. Aoki et al., Review of lattice results concerning low-energy particle physics, 1607.00299.

[8] Particle Data Group collaboration, C. Patrignani et al., Review of Particle Physics, Chin. Phys. C40 (2016) 100001. 
[9] P. Hasenfratz, M. Maggiore and F. Niedermayer, The Exact mass gap of the $O(3)$ and $O(4)$ nonlinear sigma models in $d=2$, Phys. Lett. B245 (1990) 522-528.

[10] P. Hasenfratz and F. Niedermayer, The Exact mass gap of the $O(N)$ sigma model for arbitrary $N$ is $>=$ 3 in $d=2$, Phys. Lett. B245 (1990) 529-532.

[11] P. Forgacs, F. Niedermayer and P. Weisz, The Exact mass gap of the Gross-Neveu model. 1. The Thermodynamic Bethe ansatz, Nucl. Phys. B367 (1991) 123-143.

[12] P. Hasenfratz and F. Niedermayer, The Exact correlation length of the antiferromagnetic $d=(2+1)$ Heisenberg model at low temperatures, Phys. Lett. B268 (1991) 231-235.

[13] P. Hasenfratz, The Correlation length of the Heisenberg antiferromagnet with arbitrary spin S, Eur. Phys. J. B13 (2000) 11, [cond-mat/9901355].

[14] F. Niedermayer and P. Weisz, Matching effective chiral Lagrangians with dimensional and lattice regularizations, JHEP 04 (2016) 110, [1601.00614].

[15] F. Niedermayer and P. Weisz, Massless sunset diagrams in finite asymmetric volumes, JHEP 06 (2016) 102, [1602.03159].

[16] H. Leutwyler, Energy Levels of Light Quarks Confined to a Box, Phys. Lett. B189 (1987) 197-202.

[17] P. Hasenfratz, The QCD rotator in the chiral limit, Nucl. Phys. B828 (2010) 201-214, [0909.3419].

[18] P. Hasenfratz and F. Niedermayer, Finite size and temperature effects in the AF Heisenberg model, Z. Phys. B92 (1993) 91, [hep-lat/9212022].

[19] F. Niedermayer and C. Weiermann, The rotator spectrum in the delta-regime of the $O(n)$ effective field theory in 3 and 4 dimensions, Nucl. Phys. B842 (2011) 248-263, [1006.5855].

[20] P. Hasenfratz, Lattice Quantum Chromodynamics, NATO Sci. Ser. B 122 (1985) 095.

[21] C. Pica, Beyond the Standard Model: Charting Fundamental Interactions via Lattice Simulations, PoS LATTICE2016 (2016) 015, [1701.07782].

[22] P. Hasenfratz and F. Niedermayer, Perfect lattice action for asymptotically free theories, Nucl. Phys. B414 (1994) 785-814, [hep-lat/9308004].

[23] P. Hasenfratz, F. Niedermayer and R. von Allmen, Lattice Regularization and Symmetries, JHEP 10 (2006) 010, [hep-lat/0606021].

[24] M. G. Endres, R. C. Brower, W. Detmold, K. Orginos and A. V. Pochinsky, Multiscale Monte Carlo equilibration: Pure Yang-Mills theory, Phys. Rev. D92 (2015) 114516, [1510.04675].

[25] W. Detmold and M. G. Endres, Multiscale Monte Carlo equilibration: Two-color QCD with two fermion flavors, Phys. Rev. D94 (2016) 114502, [1605.09650].

[26] M. G. Endres, New simulation strategies for lattice gauge theory, in Proceedings, 34th International Symposium on Lattice Field Theory (Lattice 2016): Southampton, UK, July 24-30, 2016, 2016. 1612.01609.

[27] P. Hasenfratz, Prospects for perfect actions, Nucl. Phys. Proc. Suppl. 63 (1998) 53-58, [hep-lat/9709110].

[28] P. H. Ginsparg and K. G. Wilson, A Remnant of Chiral Symmetry on the Lattice, Phys. Rev. D25 (1982) 2649.

[29] P. Hasenfratz, Lattice QCD without tuning, mixing and current renormalization, Nucl. Phys. B525 (1998) 401-409, [hep-lat/9802007]. 
[30] High Energy Physics (HEP) information system INSPIRE, Top Cited Articles of All Time (2016 edition) by hep-lat.

[31] P. Hasenfratz, V. Laliena and F. Niedermayer, The Index theorem in QCD with a finite cutoff, Phys. Lett. B427 (1998) 125-131, [hep-lat/9801021].

[32] H. Neuberger, More about exactly massless quarks on the lattice, Phys. Lett. B427 (1998) 353-355, [hep-lat/9801031].

[33] M. Lüscher, Exact chiral symmetry on the lattice and the Ginsparg-Wilson relation, Phys. Lett. B428 (1998) 342-345, [hep-lat/9802011].

[34] M. Lüscher, Abelian chiral gauge theories on the lattice with exact gauge invariance, Nucl. Phys. B549 (1999) 295-334, [hep-lat/9811032].

[35] H. Neuberger, Geometrical aspects of chiral anomalies in the overlap, Phys. Rev. D59 (1999) 085006, [hep-lat/9802033].

[36] Y. Kikukawa and A. Yamada, Weak coupling expansion of massless QCD with a Ginsparg-Wilson fermion and axial U(1) anomaly, Phys. Lett. B448 (1999) 265-274, [hep-lat/9806013].

[37] M. Lüscher, Topology and the axial anomaly in Abelian lattice gauge theories, Nucl. Phys. B538 (1999) 515-529, [hep-lat/9808021].

[38] T.-W. Chiu, The Axial anomaly of Ginsparg-Wilson fermion, Phys. Lett. B445 (1999) 371-377, [hep-lat/9809013].

[39] D. H. Adams, Axial anomaly and topological charge in lattice gauge theory with overlap Dirac operator, Annals Phys. 296 (2002) 131-151, [hep-lat/9812003].

[40] K. Fujikawa, A Continuum limit of the chiral Jacobian in lattice gauge theory, Nucl. Phys. $\mathbf{B 5 4 6}$ (1999) 480-494, [hep-th/9811235].

[41] H. Suzuki, Simple evaluation of chiral Jacobian with overlap Dirac operator, Prog. Theor. Phys. 102 (1999) 141-147, [hep-th/9812019].

[42] H. So and N. Ukita, Ginsparg-Wilson relation and lattice supersymmetry, Phys. Lett. B457 (1999) 314-318, [hep-lat/9812002].

[43] D. B. Kaplan, A Method for simulating chiral fermions on the lattice, Phys. Lett. B288 (1992) 342-347, [hep-lat/9206013].

[44] R. Narayanan and H. Neuberger, Infinitely many regulator fields for chiral fermions, Phys. Lett. $\mathbf{B 3 0 2}$ (1993) 62-69, [hep-lat/9212019].

[45] Y. Shamir, Chiral fermions from lattice boundaries, Nucl. Phys. B406 (1993) 90-106, [hep-lat/9303005].

[46] H. Neuberger, Exactly massless quarks on the lattice, Phys. Lett. B417 (1998) 141-144, [hep-lat/9707022].

[47] D. M. Grabowska and D. B. Kaplan, Chiral solution to the Ginsparg-Wilson equation, Phys. Rev. D94 (2016) 114504, [1610.02151].

[48] P. Hasenfratz and R. von Allmen, Towards Weyl fermions on the lattice without artefacts, JHEP 02 (2008) 079, [0710.5346]. 